\title{
Shear viscosity to electrical conductivity ratio in the quasiparticle models
}

\author{
Valeriya Mykhaylova ${ }^{a}$ \\ Institute of Theoretical Physics, University of Wrocław, PL-50204 Wrocław, Poland
}

Received 13 June 2020 / Accepted 27 October 2020

Published online 21 December 2020

\begin{abstract}
We examine the temperature dependence of the shear viscosity $\eta$ to electrical conductivity $\sigma$ ratio, as well as the specific shear viscosity and the scaled electrical conductivity in QCD with light and strange quarks. Our calculations are performed in kinetic theory under the relaxation time approximation combined with the quasiparticle model. We compute all transport parameters using the isotropic and transport cross sections and compare our results to a class of quasiparticle models for the QGP with $N_{f}=2+1$. The results depending on different schemes are examined. The ratio $(\eta / s) /(\sigma / T)$ quantifies the relation between the relaxation times of gluons and quarks and specifies their comparative role in the evolution of the QGP. We find an excellent agreement with the $(\eta / s) /(\sigma / T)$ ratio deduced from the dynamical quasiparticle model in which the quasiparticles are characterized not only by their effective masses but also by finite widths.
\end{abstract}

\section{Introduction}

Intensive studies of the experimental data obtained in ultrarelativistic heavy ion collisions confirmed that deconfined QCD matter, the quark-gluon plasma (QGP), behaves as a strongly-coupled system [1-4]. Its evolution is described by the ideal $[5,6]$ and viscous [7-10] hydrodynamics. The latter includes the dissipative phenomena occurring in the medium, whose dynamical properties are encoded in the particular transport coefficients.

One of these parameters is the shear viscosity $\eta$. It corresponds to the shear stress produced in the non-equilibrated system and quantifies the reaction of the medium to the exchange of a momentum between its elements. The shear viscosity enters the fluid dynamical simulations as a dimensionless quantity $\eta / s$, where it is scaled by the entropy density $s$. The $\eta / s$ ratio in first-principles lattice QCD (lQCD) calculations is currently available in the pure SU(3) theory only [11-15]. Gauge/gravity duality yields the well-known Kovtun-Son-Starinets (KSS) bound $1 / 4 \pi$ [16] for the specific shear viscosity. Different theoretical and phenomenological approaches have been used to examine transport properties of deconfined matter in pure Yang-Mills theory $[17,18]$, and in the presence of two light quarks [19-21]. The results consistently

a e-mail: valeriya.mykhaylova@uwr.edu.pl 
show that the $\eta / s$ ratio exhibits a minimum at the phase transition temperature, being larger than the KSS bound.

The recent studies of the specific shear viscosity of the QGP with $N_{f}=2+1$ are performed in the phenomenological models, describing QCD matter in terms of the quasiparticle excitations [22-26]. In these models, the essential physics of strong interactions is accommodated into the thermodynamics via an effective coupling and dynamically generated masses depending on temperature $T$ and chemical potential $\mu$. The validity and efficiency of the quasiparticle model (QPM) are supported by the lQCD equation of state.

Another valuable transport parameter is the longitudinal electrical conductivity $\sigma$, which quantifies the response of the QGP to the electric field produced in ultrarelativistic heavy ion collisions. The dimensionless ratio $\sigma / T$ reflects the ability of the system to conduct the longitudinal component of the electric charge current, i.e. the electric charge itself. The electrical conductivity has been evaluated in 1QCD simulations [27-31], as well as by using the Green-Kubo formalism [23,32], the AdS/CFT framework [33] and a class of quasiparticle approaches [23,24,26,32,34].

The ratio of $(\eta / s) /(\sigma / T)$ also reveals important information about the interactions between the medium constituents. It was shown in [23] that $(\eta / s) /(\sigma / T)$ complements the ratio of gluon to quark scattering rates and appears to be independent from the effective coupling. At $T_{c}$, the $(\eta / s) /(\sigma / T)$ ratio was predicted to rapidly grow, reaching the value $\approx 20$, and become approximately constant at higher temperatures, $\approx 4$. Along with the studies of the flavor dependence of $\eta / s[25,26]$ and $\sigma / T$ [35], the relation between these quantities may help us to understand the comparative role of gluons to quarks in the evolution of the QGP.

In this work, using the quasiparticle description in the relaxation time approximation, we compute the specific shear viscosity $\eta / s$, the scaled electrical conductivity $\sigma / T$ and the shear viscosity to electrical conductivity ratio, $(\eta / s) /(\sigma / T)$. We collect the corresponding results from other available quasiparticle models $[23,24,26]$ and discuss their main features, which result in quantitative differences of the computed transport parameters.

\section{Transport parameters in the quasiparticle model}

We evaluate transport parameters of the QGP from the expressions derived in kinetic theory under the relaxation time approximation [36,37]. For $N_{f}=2+1$, the shear viscosity at $\mu=0$ reads [25]

$$
\eta=\frac{2}{15 T} \sum_{i=l, s, g} \int \frac{d^{3} p}{(2 \pi)^{3}} \frac{p^{4}}{E_{i}^{2}} d_{i} \tau_{i} f_{i}^{0}\left(1 \pm f_{i}^{0}\right)
$$

where the prefactor 2 accounts for the anti-particle contributions and $d_{i}$ are the degeneracy factors: $d_{l}=2 N_{c} N_{l}=12$ for two light quarks, $d_{s}=2 N_{c}=6$ for strange quarks and $d_{g}=2\left(N_{c}^{2}-1\right)=16$ for gluons. Further, for each quasiparticle species, $\tau_{i}$ is the relaxation time and $f_{i}^{0}=\left(\exp \left(E_{i} / T\right) \pm 1\right)^{-1}$ is the corresponding statistical distribution function. The dispersion relation for massive on-shell quasiparticles reads $E_{i}=\sqrt{p^{2}+m_{i}^{2}}$, with dynamically generated temperature-dependent masses $m_{i}^{2}(T)=\left(m_{i}^{0}\right)^{2}+\Pi_{i}(T)$. We include the bare masses of light and strange quarks as $m_{l}^{0}=5 \mathrm{MeV}$ and $m_{s}^{0}=95 \mathrm{MeV}$, respectively. For $\Pi_{i}(T)$ we use the asymptotic forms 
of the gauge-independent hard thermal loop (HTL) self-energies [38,39],

$$
\begin{aligned}
\Pi_{g}(T) & =\left(3+\frac{N_{f}}{2}\right) \frac{G(T)^{2}}{6} T^{2}, \\
\Pi_{l, s}(T) & =2\left(m_{l, s}^{0} \sqrt{\frac{G(T)^{2}}{6} T^{2}}+\frac{G(T)^{2}}{6} T^{2}\right),
\end{aligned}
$$

where the perturbative running coupling has been replaced with an effective coupling $G(T)$ deduced from the lQCD entropy density [40,41]. The numerical results for the coupling and masses have been presented in [25].

In the relaxation time approximation, the electrical conductivity at $\mu=0$ is expressed as [24]

$$
\sigma=\frac{2}{3 T} \sum_{i=u, d, s} \int \frac{d^{3} p}{(2 \pi)^{3}} \frac{p^{2}}{E_{i}^{2}} q_{i}^{2} d_{i} \tau_{i} f_{i}^{0}\left(1-f_{i}^{0}\right)
$$

Here, all quark contributions are evaluated separately due to the different electric charges $q_{i}$. Explicitly, $q_{u}=2 e / 3$ for up quarks and $q_{d, s}=-e / 3$ for down and strange quarks. The electron charge $e=(4 \pi \alpha)^{1 / 2}$ is expressed through the fine structure constant, $\alpha \simeq 1 / 137$. For gluons, $q_{g}=0$, therefore they do not contribute to the electrical conductivity. Note that, for each term of the sum, the degeneracy factor is taken as $d_{u, d, s}=6$, which differs from our convention for the shear viscosity given in equation (1).

\subsection{Relaxation time}

The relaxation time $\tau_{i}$ is a parameter of the approximate solution to the Boltzmann equation $[42,43]$, and is defined as

$$
\tau_{i}=\left(n_{i} \bar{\sigma}_{i}\right)^{-1}
$$

where $n_{i}$ is the equilibrium number density and $\bar{\sigma}_{i}$ is the energy-averaged total cross section. It includes the averaged cross sections of the considered two-body elementary scatterings among the quasiparticles.

Following $[19,44]$, we calculate the individual thermal-averaged transport cross section using

$$
\bar{\sigma}_{t r}(T)=\int d s \int d t \frac{d \sigma_{i j \rightarrow i^{\prime} j^{\prime}}}{d t} \sin ^{2} \theta\left(s, t, m_{i, j, i^{\prime}, j^{\prime}}(T)\right)\left(1 \pm f_{i^{\prime}}^{0}\right)\left(1 \pm f_{j^{\prime}}^{0}\right) P(s ; T),
$$

where the differential cross section $d \sigma_{i j \rightarrow i^{\prime} j^{\prime}} / d t$ is evaluated at tree level using the standard prescription in the center-of-mass frame, $s$ and $t$ are Mandelstam variables. The $\left(1 \pm f_{i^{\prime}, j^{\prime}}^{0}\right)$ is the Pauli blocking $(+)$ or Bose enhancement $(-)$ factor which corresponds to the medium effects and $P(s ; T)$ denotes the probability of obtaining the final-state particles with the total energy $s$. In equation (6), each component includes the effective masses of the quasiparticles. More details on the transport cross sections are found in [25]. Now we would like to focus on the phenomenological scaling factor $\sin ^{2} \theta\left(s, t, m_{i, j, i^{\prime}, j^{\prime}}(T)\right)$ derived from the kinematics of the processes $[19,44-46]$. Here, $\theta$ is the scattering angle between the two initial particles, and $m_{i, j, i^{\prime}, j^{\prime}}(T)$ are the effective masses of the scattering participants. 
The weight factor $\sin ^{2} \theta\left(s, t, m_{i, j, i^{\prime}, j^{\prime}}(T)\right)$ is introduced to exclude the scattering processes with the small initial angle, leading to the processes with the large angle $\theta$ prevailing in the momentum transfer between the medium constituents. Thus, $\bar{\sigma}_{t r}(T)$ brings the major impact to the transport coefficients of the QCD medium.

Nevertheless, we additionally evaluate the transport parameters without the large angle scattering approximation, by setting $\sin ^{2} \theta\left(s, t, m_{i, j, i^{\prime}, j^{\prime}}(T)\right)=1$ in equation (6). In this way, we get the total energy-averaged cross section in the isotropic limit, $\sigma_{i s o}(T)$. The cross sections are approximately $\bar{\sigma}_{\text {iso }}(T) \approx 2 \bar{\sigma}_{t r}(T) / 3$. The numerical results for the shear viscosity and electrical conductivity using different types of the cross sections are presented in Sections 3.1, 3.2, and 3.3.

\section{Discussion and numerical results}

Before we compare our outcomes to the other approaches, let us briefly highlight their key elements: a variety of definitions for the effective coupling and masses as well as different treatments of the cross sections lead to somewhat different behaviors of the transport parameters.

In the pioneering work presenting the $(\eta / s) /(\sigma / T)$ ratio [23], the shear viscosity and the electrical conductivity were obtained from the Green-Kubo formulas. The thermodynamics of that quasiparticle model is based on the IQCD data as in our QPM [40,41], although their effective coupling was determined by fitting the energy density in lQCD, whereas in our QPM by the entropy density, to avoid a complication of the bag function. In addition, while gluon effective masses share the same expression, equation (2), the effective quark mass obeys a different form and does not depend on the current mass $m_{i}^{0}, m_{q}^{2}=G^{2}(T) T^{2} / 3$. The relaxation times are evaluated using the parametric transport cross sections of the form [23]

$$
\sigma_{t r}^{i j}(s)=\beta^{i j} \frac{\pi \alpha_{s}^{2}}{m_{D}^{2}} \frac{s}{s+m_{D}^{2}} h(a),
$$

where $\beta^{i j}$ is the coefficient referring to the different interactions between quarks and gluons, $\beta^{q q}=16 / 9, \beta^{q q^{\prime}}=8 / 9, \beta^{q g}=2, \beta^{g g}=9$. The Debye mass $m_{D}^{2}=G^{2}(T) T^{2}$ is originated from the HTL approach, while $\alpha_{s}=G^{2} / 4 \pi$ is the strong coupling and $h(a)=4 a(1+a)[(2 a+1) \ln (1+1 / a)-2]$ with $a=m_{D}^{2} / s$ represents anisotropy of the scatterings [23].

The $(\eta / s) /(\sigma / T)$ ratio is also studied in a slightly different quasiparticle model [24], employing equations (1) and (4) for $\eta$ and $\sigma$, respectively, while the relaxation times for massless quarks and gluons were used [36],

$$
\begin{aligned}
\tau_{q(\bar{q})} & =\left[5.1\left(1+0.12\left(2 N_{f}+1\right)\right) \alpha_{s} \log \alpha_{s}^{-1}\right]^{-1}, \\
\tau_{g} & =\left[22.5\left(1+0.06 N_{f}\right) \alpha_{s} \log \alpha_{s}^{-1}\right]^{-1} .
\end{aligned}
$$

At $\mu=0$, the effective masses read $m_{i}^{2}=\left(m_{i}^{0}\right)^{2}+m_{i}^{0} G(T) T / \sqrt{3}+G^{2}(T) T^{2} / 6$, with the bare masses $m_{l}^{0}=8 \mathrm{MeV}$ for light quark, $m_{s}^{0}=80 \mathrm{MeV}$ for strange quark and $m_{g}^{0}=0$ for gluons. The QCD running coupling $G(T)$ is expanded up to the two-loop order using the lQCD data from [47].

Finally, we briefly comment on the dynamical quasiparticle model (DQPM) [26] and its thermodynamics based on $[40,41]$. In this approach, for vanishing chemical potential, the transport parameters are evaluated using equations (1) and (4). The 
effective coupling is then determined by the 1QCD entropy density [40,41], as done for our effective coupling. The dynamical masses of quarks and gluons obey

$$
\begin{aligned}
& M_{q}^{2}(T)=\frac{N_{c}^{2}-1}{8 N_{c}} G^{2}(T) T^{2}, \\
& M_{g}^{2}(T)=\left(N_{c}+\frac{1}{2} N_{f}\right) \frac{G^{2}(T)}{6} T^{2} .
\end{aligned}
$$

The heavier strange quarks are distinguished from the light quarks through the relation $M_{s}(T)=M_{u}+\Delta M=M_{d}+\Delta M$ with $\Delta M=30 \mathrm{MeV}$. Additionally, the DQPM incorporates the finite widths of the quasiparticles [26]. The relaxation time is defined as an inverse of the momentum-dependent interaction rate $\Gamma_{i}^{o n}\left(\mathbf{p}_{i}, T\right)$ [48],

$$
\Gamma_{i}^{o n}\left(\mathbf{p}_{i}, T\right)=\sum_{j=q, \bar{q}, g} \int \frac{d^{3} p_{j}}{(2 \pi)^{3}} d_{j} f_{j} v_{r e l} \int d \sigma_{i j \rightarrow i^{\prime} j^{\prime}}^{o n}\left(1 \pm f_{i^{\prime}}\right)\left(1 \pm f_{j^{\prime}}\right)
$$

where the superscript on stands for the on-shell quasiparticles. The relative velocity $v_{r e l}$ between the colliding particles in our expression is inserted into the probability $P(s ; T)$, see equation (6). One may notice that the phenomenological weight factor $\sin ^{2} \theta\left(s, t, m_{i, j, i^{\prime}, j^{\prime}}(T)\right)$ is not included in equation (12).

In the following subsections, we present the specific shear viscosity $\eta / s$, the scaled electrical conductivity $\sigma / T$ and the shear viscosity to electrical conductivity ratio $(\eta / s) /(\sigma / T)$ obtained at $\mu=0$ in the current quasiparticle model and the approaches discussed above.

\subsection{Specific shear viscosity}

Figure 1 shows the shear viscosity to entropy density ratio $\eta / s$ of the QGP for $N_{f}=2+1$ computed in various quasiparticle models. In all approaches, the specific shear viscosity exhibits a shallow minimum around the pseudo-critical temperature and increases monotonically at higher temperatures. We observe that in the quasiparticle models, for the QGP with massive quarks, the ratio does not reach the lower bound $\eta / s=1 / 4 \pi \simeq 0.08$, conjectured in [16] in the gravity dual to conformal gauge theories, unless the relaxation time is tuned so that $\eta / s$ approaches the limit, as done in [22]. For temperatures above $1.5 T_{c}$, the curves seem to evolve with approximately equal slopes shifted by different constants, so that the possible values of $\eta / s$ vary within one order of magnitude.

In comparison to the specific shear viscosity obtained from the transport cross sections $\bar{\sigma}_{t r}$, one finds an overall downward shift of the ratio calculated in the isotropic limit employing $\bar{\sigma}_{i s o}$. The almost constant difference between the cross sections, $\bar{\sigma}_{i s o} \approx 2 \bar{\sigma}_{t r} / 3$, leads to the discrepancy between the $\eta / s$ curves.

Further, despite the different definitions of the cross sections, we observe a qualitative agreement between our results and other quasiparticle models. The specific shear viscosity based on the transport cross sections is consistent with $\eta / s$ found within the QPM by Puglisi et al. [23]. When the isotropic cross sections are implemented into the shear viscosity, our QPM result is in good agreement with that in the DQPM [26]. Additionally, at $T_{c}$, our isotropic result is close to $\eta / s$ computed in the QPM by Thakur et al. [24], where the relaxation times for massless quarks and gluons were used, although at high $T$, the outcome of this assumption reaches noticeably smaller values when compared to the other curves. 


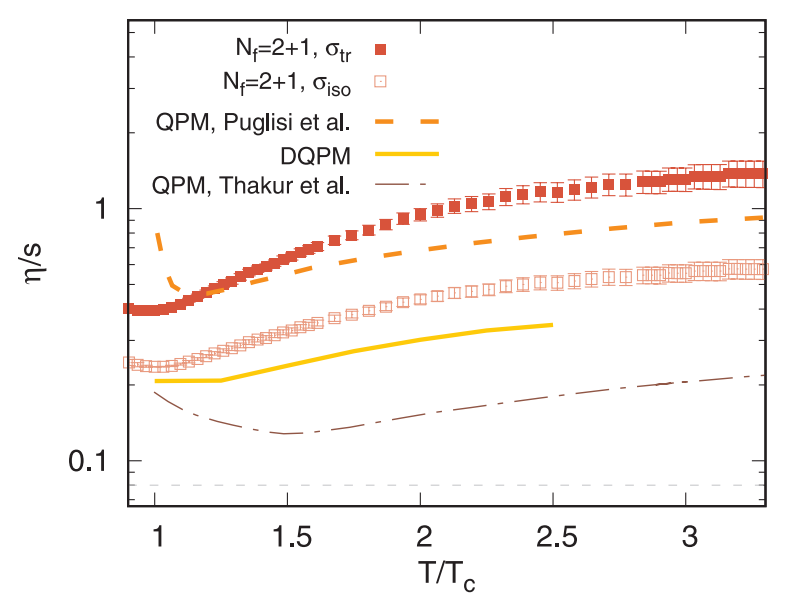

Fig. 1. Shear viscosity to entropy density ratio as a function of $T / T_{c}$ in various quasiparticle approaches. Our results are obtained with the transport cross sections (full squares), as well as in the isotropic limit (open squares). For comparison, we present the $\eta / s$ ratio obtained in other QPM [23] (dashed line), DQPM [26] (solid line) and QPM with the relaxation times for massless particles [24] (dash-dotted line). The horizontal dashed line indicates the KSS-bound of $1 /(4 \pi) \approx 0.08$.

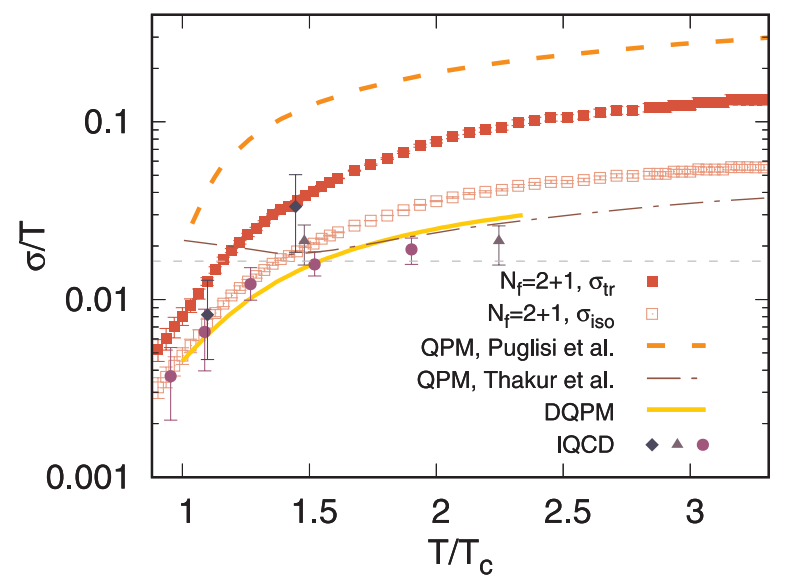

Fig. 2. Electrical conductivity to temperature ratio as a function of $T / T_{c}$ calculated in the current QPM with transport cross sections (full squares) and isotropic cross sections (open squares). Additionally, the results presented in [23] (dashed line), [24] (dash-dotted line), and in [26] (solid line) are shown along with the lQCD data from [28] (diamonds), [29] (triangles) and [30,31] (circles). The horizontal dashed line indicates the value $\sigma / T=e^{2} N_{c}^{2} /(16 \pi) \approx 0.017$ found in AdS/CFT approach [33].

\subsection{Electrical conductivity}

In Figure 2, we compare the electrical conductivity to temperature ratio $\sigma / T$ computed in various quasiparticle approaches. Additionally, we present the AdS/CFT result, $\sigma / T=e^{2} N_{c}^{2} /(16 \pi) \approx 0.017$ [33], and the available lQCD data for $N_{f}=2+1$.

In the isotropic limit, our result is consistent with the electrical conductivity obtained in the DQPM [26] and in the QPM by Thakur et al. [24] (at $T \geq 1.5 T_{c}$ ). Also, near $T_{c}$, our isotropic result coincides within the errors with the IQCD 


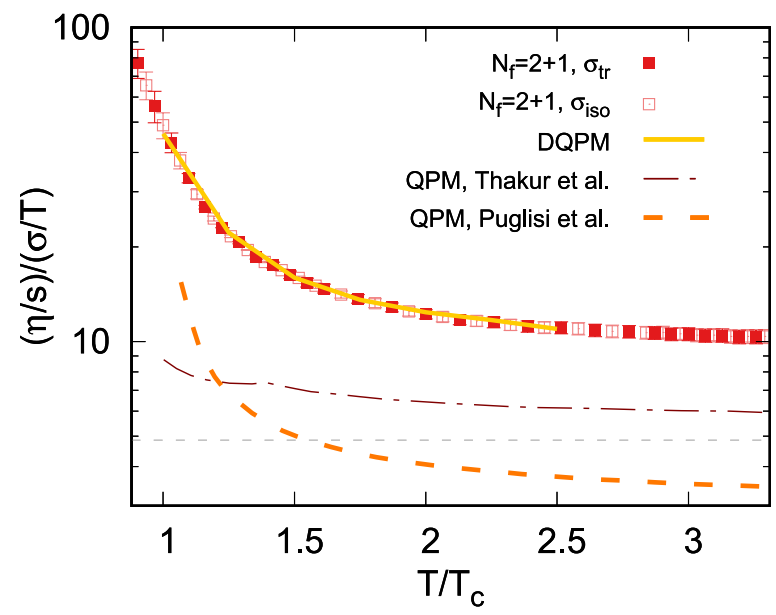

Fig. 3. The ratio of the shear viscosity to the electrical conductivity as a function of $T / T_{c}$ obtained in different QPM approaches. The results from our quasiparticle model with transport (full squares) and isotropic (open squares) cross sections are presented along with the DQPM [26] and the quasiparticle models by [24] (dash-dotted line) and [23] (dashed curve). The horizontal dashed line indicates the value $(\eta / s) /(\sigma / T)=4 /\left(e^{2} N_{c}^{2}\right) \approx 4.85$ obtained from the AdS/CFT results [16,33].

data [28-31]. The electrical conductivity calculated from the transport cross sections also agrees with the lQCD data [28] and around $T_{c}$ appears quite close to the isotropic result and to the DQPM [26]. In the studied temperature range, $\sigma / T$ evaluated in the QPM by Puglisi et al. [23] reaches much higher values than in any other effective approaches. It appears to be nearly two times bigger than $\sigma / T$ calculated in our QPM using transport cross sections. This observation differs from the specific shear viscosity, with which we have a nice agreement between the approach in [23] and ours based on the transport cross sections.

As discussed earlier in this section, the QPM by Puglisi et al. [23] and the DQPM [26] are based on the common 1QCD equation of state [40,41], same as in the current QPM. However, the setup by Thakur et al. [24] is completely different and strongly affects the behavior of $\sigma / T$ near $T_{c}$. All the other curves grow with the temperature, while $\sigma / T$ presented in [24] exhibits a mild minimum at $T=1.5 T_{c}$, same as the specific shear viscosity evaluated in their model, see Figure 1.

\subsection{Shear viscosity to electrical conductivity ratio}

Figure 3 exhibits the temperature dependence of the $(\eta / s) /(\sigma / T)$ ratio, obtained from $\eta / s$ presented in Figure 1 and $\sigma / T$ shown in Figure 2.

We find an excellent agreement between and the DQPM [26] and the current QPM with either transport or isotropic cross sections applied. The discrepancies in $\eta / s$ and $\sigma / T$ computed in these models are now canceled out. From equations (1) and (4) it follows that the electrical conductivity depends on the relaxation times of quarks, while the shear viscosity contains an additional term created by the presence of gluons. Therefore, the quantity $(\eta / s) /(\sigma / T)$ depends on the ratio of the gluon relaxation times to the relaxation times of light and strange quarks. The shear viscosity also differs from the electrical conductivity by its temperature dependence, which among other terms, is encoded in the ratio $p^{4} / E_{i}^{2}$ in equation (1), and $p^{2} / E_{i}^{2}$ in equation (4). Since $\eta$ and $\sigma$ equivalently depend on the effective coupling and masses, we expect 
that the influence of these details to a large extent cancels in the $(\eta / s) /(\sigma / T)$ ratio. As a result, the shear viscosity to electrical conductivity remains sensitive only to the ratio $\tau_{g} /\left(\tau_{l}+\tau_{s}\right)$. This observation agrees with the conclusion in [23], although the same relaxation times for all types of quarks were assumed, unlike in our approach to distinguish the strange from light quarks.

Our explicit study justifies that the influence of the effective coupling and masses is compensated. We also find a similar cancellation mechanism of the finite widths of quasiparticles in the $(\eta / s) /(\sigma / T)$ ratio. The observed agreement can be traced back to the relaxation times, modeled in a very similar fashion and depending on the same types of the two-body scattering processes $[25,48]$. Thus, both approaches equivalently maintain the microscopic interactions between the constituents of deconfined QCD matter.

On the other hand, the $(\eta / s) /(\sigma / T)$ ratio calculated in the quasiparticle model by Puglisi et al. [23] depends on the pQCD-type cross sections, see equation (7), and the same relaxation times for all quark flavors. At the pseudo-critical temperature, the ratio amounts to about 20, while our result, together with the DQPM, yields at $T_{c}$ more than two times bigger value, $(\eta / s) /(\sigma / T) \approx 50$. We recall that our specific shear viscosity based on the transport cross sections appears close to the one found in [23], yet their larger value of $\sigma / T$ significantly diminishes the $(\eta / s) /(\sigma / T)$ ratio evaluated in that method.

The shear viscosity to electrical conductivity ratio obtained in the model by Thakur et al. [24] is the least sensitive to the temperature changes. Compared to the other approaches, it remains approximately constant in the explored temperature range, exhibiting a slight increase near $T_{c}$.

In general, the $(\eta / s) /(\sigma / T)$ ratio in the quasiparticle models shows a pronounced increase toward the pseudo-critical temperature and is expected to reach an approximately constant value at higher temperatures. In contrast, from the AdS/CFT predictions for $\eta / s[16]$ and $\sigma / T$ [33], one obtains $(\eta / s) /(\sigma / T)=4 /\left(e^{2} N_{c}^{2}\right) \approx 4.85$ at any temperature.

\section{Conclusions}

In this work, we investigated the realistic strongly interacting system with $N_{f}=2+1$ quark flavors and analyzed its transport parameters using the isotropic and effective cross sections. While it is expected that the cross sections with the large angle scattering approximation dominate the transport properties of the medium, in the isotropic limit, we found a considerable suppression of the shear viscosity and electrical conductivity.

We compared our results to the coefficients evaluated in various quasiparticle models, and carefully examined the potential origins of qualitative and quantitative differences among them.

We observed that the shear viscosity to electrical conductivity ratio compensates the details of the effective coupling and masses, but preserves the overall dependence on the relation between the relaxation times of gluons and quarks. Therefore, the $(\eta / s) /(\sigma / T)$ ratio is a valuable quantity to compare the role of quarks and gluons in various effective models. In fact, we found a remarkable agreement with the $(\eta / s) /(\sigma / T)$ ratio deduced from the results of the DQPM. The observed consistency justifies that both approaches identically accommodate the microscopic interactions between the QGP constituents, despite somewhat different treatments of modeling the details.

This work was done in collaboration with Chihiro Sasaki, Krzysztof Redlich, and Marcus Bluhm. I also acknowledge helpful comments from Pok Man Lo and Michał Marczenko. The 
work was partly supported by the Polish National Science Center (NCN) under the Opus grant no. 2018/31/B/ST2/01663.

Open Access This is an open access article distributed under the terms of the Creative Commons Attribution License (https://creativecommons.org/licenses/by/4.0), which permits unrestricted use, distribution, and reproduction in any medium, provided the original work is properly cited.

Publisher's Note The EPJ Publishers remain neutral with regard to jurisdictional claims in published maps and institutional affiliations.

\section{References}

1. P. Huovinen, P. Kolb, U.W. Heinz, P. Ruuskanen, S. Voloshin, Phys. Lett. B 503, 58 (2001)

2. T. Hirano, K. Tsuda, Phys. Rev. C 66, 054905 (2002)

3. K. Dusling, D. Teaney, Phys. Rev. C 77, 034905 (2008)

4. E. Shuryak, Prog. Part. Nucl. Phys. 62, 48 (2009)

5. W. Broniowski, M. Chojnacki, W. Florkowski, A. Kisiel, Phys. Rev. Lett. 101, 022301 (2008)

6. B. Schenke, S. Jeon, C. Gale, Phys. Rev. C 82, 014903 (2010)

7. L.P. Csernai, J. Kapusta, L.D. McLerran, Phys. Rev. Lett. 97, 152303 (2006)

8. P. Romatschke, U. Romatschke, Phys. Rev. Lett. 99, 172301 (2007)

9. W. Florkowski, A. Jaiswal, E. Maksymiuk, R. Ryblewski, M. Strickland, Phys. Rev. C 91, 054907 (2015)

10. L. Du, U. Heinz, Comput. Phys. Commun. 251, 107090 (2020)

11. A. Nakamura, S. Sakai, Phys. Rev. Lett. 94, 072305 (2005)

12. H.B. Meyer, Phys. Rev. D 76, 101701 (2007)

13. H.B. Meyer, Nucl. Phys. A 830, 641C (2009)

14. S.W. Mages, S. Borsányi, Z. Fodor, A. Schäfer, K. Szabó, PoS Lattice 2014, 232 (2015)

15. N. Astrakhantsev, V. Braguta, A. Kotov, JHEP 1704, 101 (2017)

16. P. Kovtun, D.T. Son, A.O. Starinets, Phys. Rev. Lett. 94, 111601 (2005)

17. M. Bluhm, B. Kämpfer, K. Redlich, Phys. Rev. C 84, 025201 (2011)

18. N. Christiansen, M. Haas, J.M. Pawlowski, N. Strodthoff, Phys. Rev. Lett. 115, 112002 (2015)

19. C. Sasaki, K. Redlich, Nucl. Phys. A 832, 62 (2010)

20. A. Harutyunyan, D.H. Rischke, A. Sedrakian, Phys. Rev. D 95, 114021 (2017)

21. P. Singha, A. Abhishek, G. Kadam, S. Ghosh, H. Mishra, J. Phys. G 46, 015201 (2019)

22. S. Plumari, W.M. Alberico, V. Greco, C. Ratti, Phys. Rev. D 84, 094004 (2011)

23. A. Puglisi, S. Plumari, V. Greco, Phys. Lett. B 751, 326 (2015)

24. L. Thakur, P.K. Srivastava, G.P. Kadam, M. George, H. Mishra, Phys. Rev. D 95, 096009 (2017)

25. V. Mykhaylova, M. Bluhm, K. Redlich, C. Sasaki, Phys. Rev. D 100, 034002 (2019)

26. O. Soloveva, P. Moreau, E. Bratkovskaya, Phys. Rev. C 101, 045203 (2020)

27. S. Gupta, Phys. Lett. B 597, 57 (2004)

28. O. Kaczmarek, E. Laermann, M. Müller, F. Karsch, H.T. Ding, S. Mukherjee, A. Francis, W. Soeldner, PoS ConfinementX, 185 (2012)

29. G. Aarts, C. Allton, J. Foley, S. Hands, S. Kim, Phys. Rev. Lett. 99, 022002 (2007)

30. A. Amato, G. Aarts, C. Allton, P. Giudice, S. Hands, J.I. Skullerud, Phys. Rev. Lett. 111, 172001 (2013)

31. G. Aarts, C. Allton, A. Amato, P. Giudice, S. Hands, J.I. Skullerud, JHEP 02, 186 (2015)

32. A. Puglisi, S. Plumari, V. Greco, Phys. Rev. D 90, 114009 (2014)

33. S. Caron-Huot, P. Kovtun, G.D. Moore, A. Starinets, L.G. Yaffe, JHEP 12, 015 (2006) 
34. W. Cassing, O. Linnyk, T. Steinert, V. Ozvenchuk, Phys. Rev. Lett. 110, 182301 (2013)

35. V. Mykhaylova, C. Sasaki, to appear

36. A. Hosoya, K. Kajantie, Nucl. Phys. B 250, 666 (1985)

37. W. Florkowski, E. Maksymiuk, R. Ryblewski, Phys. Rev. C 97, 024915 (2018)

38. R.D. Pisarski, Nucl. Phys. A 498, 423C (1989)

39. M. Bluhm, B. Kämpfer, R. Schulze, D. Seipt, Eur. Phys. J. C 49, 205 (2007)

40. S. Borsanyi, G. Endrodi, Z. Fodor, S. Katz, S. Krieg, C. Ratti, K. Szabo, JHEP 08, $053(2012)$

41. S. Borsanyi, Z. Fodor, C. Hoelbling, S.D. Katz, S. Krieg, K.K. Szabo, Phys. Lett. B 730, 99 (2014)

42. P. Bhatnagar, E. Gross, M. Krook, Phys. Rev. 94, 511 (1954)

43. M. Bluhm, B. Kämpfer, K. Redlich, Acta Phys. Polon. Supp. 5, 619 (2012)

44. P. Zhuang, J. Hufner, S.P. Klevansky, L. Neise, Phys. Rev. D 51, 3728 (1995)

45. M. Thoma, Phys. Rev. D 49, 451 (1994)

46. S. Mitra, V. Chandra, Phys. Rev. D 94, 034025 (2016)

47. G. Boyd, J. Engels, F. Karsch, E. Laermann, C. Legeland, M. Lutgemeier, B. Petersson, Nucl. Phys. B 469, 419 (1996)

48. P. Moreau, O. Soloveva, L. Oliva, T. Song, W. Cassing, E. Bratkovskaya, Phys. Rev. C 100, 014911 (2019) 